\title{
TRANSPORT IN QUANTUM STRUCTURES OF DILUTED MAGNETIC SEMICONDUCTORS
}

\author{
J. JAROSZYŃSKI \\ Institute of Physics, Polish Academy of Sciences \\ Al. Lotników 32/46, 02-668 Warszawa, Poland
}

\begin{abstract}
This paper reviews recent millikelvin studies of magnetoconductance and noise in nanostructures of a diluted magnetic semiconductor $n-\mathrm{Cd}_{1-x} \mathrm{Mn}_{x} \mathrm{Te}$. These studies were particularly rewarding for probing the influence of magnetic ions upon mesoscopic phenomena. The accumulated results demonstrated the existence of a new driving mechanism of the universal conductance fluctuations in magnetic systems. Several signatures of spin-glass freezing were observed, such as the appearance of $1 / f$ conductance noise, aging, thermal, and magnetic irreversibilities as well as a strong increase in the amplitude of both conductance fluctuations and noise when temperature and the magnetic field were lowered below the freezing line. A statistical analysis of conductance noise made it possible to investigate the nature of excitations in spin-glass phase, and to discriminate between competing theoretical models.
\end{abstract}

PACS numbers: 73.50.Td, 72.15.Rn, 73.61.Ga, 75.50.Lk

\section{Introduction}

Changing the dimensionality of semiconductor structures allows one to examine various aspects of charge transport. Usually, we distinguish three regimes of transport: ballistic, mesoscopic, and classical. One deals with ballistic transport if all sample dimensions $L_{i}$ are shorter than the mean free path $\ell$, which is the average distance the electron travels before its initial momentum relaxes. It is of the order of the distance between impurities, which scatter electrons without changing the phases of their wave functions. The mesoscopic regime is achieved when the sample dimensions are longer than $\ell$, but shorter than $L_{\phi}>L_{i}>\ell$, as it is shown in Fig. 1, where $L_{\phi}$ is a mean distance electron passes between those of scattering processes, which destroy the phase of the electron wave, e.g. collisions with phonons, other electrons, spin-flip scattering, etc. In the classical limit all geometrical dimensions are much larger than both $L_{\phi}$ and $\ell$.

Although in the mesoscopic regime, which is of our primary interest here, we deal with diffusive charge transport, i.e. the carrier makes random walk being scattered several times from impurities, the phase of partial electron waves is preserved. 


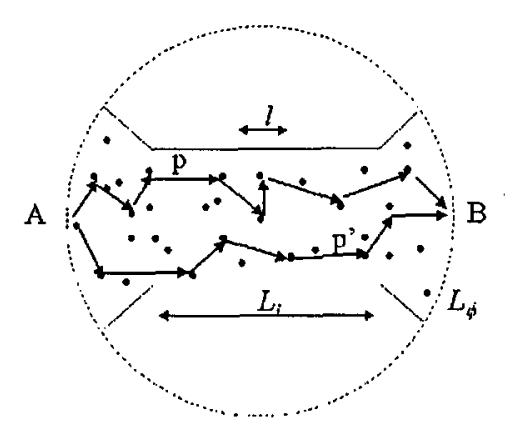

Fig. 1. Schematic view of a mesoscopic conductor. Electrons experience elastic scattering events on their way from the current source to the current sink.

Phase coherence makes quantum interference possible and accounts for the existence of several mesoscopic transport phenomena [1], such as the Aharonov-Bohm effect and universal conductance fluctuations (UCF).

The conductance $G$ is proportional to the quantum-mechanical transmission probability which, in turn, can be described in terms of interference between classical Feynman paths through the sample,

$$
G \propto P_{\mathrm{AB}}=\left|\mathrm{a}_{\mathrm{p}}+\mathrm{a}_{\mathrm{p}^{\prime}}\right|^{2}=\left|\mathrm{a}_{\mathrm{p}}\right|^{2}+\left|\mathrm{a}_{\mathrm{p}^{\prime}}\right|^{2}+2 \mathrm{a}_{\mathrm{p}} \mathrm{a}_{\mathrm{p}^{\prime}}^{*} \cos \left(\varphi_{\mathrm{p}}-\varphi_{\mathrm{p}^{\prime}}\right)
$$

where $a_{p}$ - transmission amplitude along path $p$. In classical limit, the last, interference term can be neglected, since in macroscopic conductor relative phases between different paths assume random values and the interference term averages to zero. In an external magnetic field $B=\operatorname{curl} A$, where $A$ is the vector potential, electron wave function picks up a quantum-mechanical phase,

$$
\varphi=\frac{e}{\hbar} \int_{C} A(r) \mathrm{d} r
$$

during the motion along classical path $C$ that connects two points in space. Thus, the total phase difference between two paths is given by

$$
\Delta \varphi=k_{\mathrm{F}}\left(p-p^{\prime}\right)+\frac{B S}{\hbar / e},
$$

where $k_{\mathrm{F}}$ is the Fermi wave vector and $S$ is the area enclosed between the paths. It follows from (3) that by changing the external magnetic field, the electron wavelength or the distribution of scattering centers, one can change the interference term. According to (1) this leads to sample specific nonperiodic but reproducible fluctuations of the conductance $G$ as a function of $B$. It should be emphasized that because of the interference origin of this phenomenon, $G$ is extremely sensitive to the actual shape of scattering potential. Conductance fluctuations possess one more very interesting aspect. They are called universal due to the fact that their mean square root amplitude is universal, as it was shown by a diagrammatic calculation $[2,3]$,

$$
\operatorname{rms} \Delta G=\left\langle G^{2}\right\rangle-\langle G\rangle^{2}=C \frac{e^{2}}{h}
$$

where $C$ is a numerical constant of the order of unity (actually its value depends only on the universality class, i.e. symmetry of the system). 
Another striking peculiarity of UCF is their extreme sensitivity to the changes in the scattering potential. Although mesoscopic samples contain typically $10^{19}$ atoms, under appropriate conditions the conductance change induced by moving a single scattering center can be as large as that produced by changing all the centers in the entire sample. This result can be understood by a simple physical argument. For a disordered material the Feynman paths are random walks with step size $\ell$. The number of sites visited by each Feynman path that crosses the sample is $(L / \ell)^{2}$. The total number of scattering sites in the sample is $(L / \ell)^{d}$, where $d$ is dimensionality of the system. In one-dimensional sample this means that each Feynman path actually visits a given site many times, so that the motion of single scatterer on the distance of electron wavelength can accumulate sufficient phase shift to change the conductance by $e^{2} / h$ [4].

The phenomenon of UCF is a beautiful illustration of the fundamental question of quantum mechanics, namely which path an electron actually chooses in an interference experiment. If the answer were known, the interference image would disappear as a consequence of the Heisenberg uncertainty principle [5]. Now, the path would be known when the passing electron changes the environment energy. When, for instance, electron scatters from magnetic ion with an inelastic spin-flip process, we can tell which path was actually chosen by the carrier, looking at the spin configuration. This means that the dephasing process can be regarded as a consequence of the uncertainty principle.

This article reviews the recent studies $[6,7]$ of electron transport phenomena in nanostructures of a diluted magnetic semiconductor [8] $n-\mathrm{Cd}_{1-x} \mathrm{Mn}_{x}$ Te doped either with indium or iodine. The II-VI diluted magnetic semiconductors (DMS) offer a unique opportunity to examine the influence of spin effects upon quantum transport phenomena. This is because the independent control of the carrier concentration and the incorporation of the transition metals into the elemental or III-V semiconductors is not as easy, if possible at all, as to II-VI compounds. We discovered a new driving mechanism of UCF by external magnetic field in quantum wires of $n-\mathrm{Cd}_{1-x} \mathrm{Mn}_{x} \mathrm{Te}(0 \leq x \leq 0.20)$. Usually, the external magnetic field induces the conductance fluctuations by changing the relative phase of the interfering electron waves. Our results demonstrate that there is another effect of $B$ in magnetic systems. Due to the exchange interaction between the localized spins and the carriers a giant spin-splitting of the bands occurs. Its magnitude can be of the order of the Fermi energy. This leads to a redistribution of the carriers between the spin subbands and to a substantial change of the wavelength of the electrons at the Fermi level. According to Eq. (1) this induces the conductance fluctuations.

At the same time, DMS are particularly suitable for the meaningful examination of the spin-glass phase [9] by the phenomena of coherent transport. Despite many years of considerable experimental and theoretical effort, a satisfactory understanding of the magnetic order in spin glasses is still lacking. In DMS, the localized spins are coupled by short-range antiferromagnetic superexchange interactions, which in the studied range of the $\mathrm{Mn}$ concentrations, $0.01 \leq x \leq 0.2$, lead to the spin-glass transition at $0.01 \leq T_{\mathrm{g}} \leq 2.2 \mathrm{~K}$, respectively [10]. 
It should be emphasized that by means of coherent transport we can study very small spin-glass samples, in which nontrivial statistics of excitations may be expected. Their meaningful measurements by other method such as SQUID magnetometry would be impossible. It is now well established that substantial magnetization noise is present in spin glasses. This noise is due to slow dynamics characterizing spin-glass phase, and can be detected by SQUID magnetometry in sufficiently large samples. Its power spectrum is inversely proportional to frequency, and such $1 / f$ noise is actually expected for systems with a wide spectrum of thermal excitations. It was suggested by Altshuler and Spivak [11] and Feng et al. [12] that the sensitivity of quantum interference of scattered waves to the instantaneous configuration of the localized spins in mesoscopic systems might serve as an important tool for the observation of spin-glass dynamics. Since then considerable efforts have been devoted to studies of metallic nanostructures doped with magnetic impurities. In particular, Weissman et al. [13], by employing high current density, detected $1 / f$ conductance noise in mesoscopic wires of $\mathrm{Cu}_{1-x} \mathrm{Mn}_{x}$. Very recently Neuttiens et al. [14] reported on $1 / f$ noise in wires of $A u F e$ spin glass. Despite this progress, the picture emerging from the studies of mesoscopic metallic spin-glass systems is rather confusing [15].

Our results corroborate the existence of $1 / f$ electric noise, detected here down to $30 \mathrm{mK}$. At the same time, we observe other signatures of the spin-glass freezing such as aging, thermal and magnetic irreversibilities, as well as a strong increase in the amplitude of both UCF and the noise when the temperature and the magnetic field are reduced below the freezing line. We demonstrate, by analysing non-Gaussian statistics that the data for DMS are consistent with the droplet model [16] of short-range spin glasses as well as with qualitative predictions of Monte Carlo simulations of conductance in the Edwards-Anderson model of spin glasses [17].

\section{Samples}

Our $\mathrm{Cd}_{1-x} \mathrm{Mn}_{x} \mathrm{Te}(0 \leq x \leq 0.20)$ films, having thickness of $0.3 \mu \mathrm{m}$, and electron concentrations $n$ up to $10^{19} \mathrm{~cm}^{-3}$ were grown by molecular beam epitaxy (MBE) on semi-insulating (SI) GaAs with $10 \AA \mathrm{ZnTe}$ and $3 \mu \mathrm{m} \mathrm{CdTe}$ undoped buffer layers. They were doped with either indium or iodine. The wires were fabricated by means of electron-beam lithography, followed by wet etching in $0.05 \%$ solution of $\mathrm{Br}_{2}$ in ethylene glycol. Typically, they have a mean width of $W=0.3 \mu \mathrm{m}$. For magnetoresistance measurement we used typical Hall bar geometry, while for the studies of conductance noise the arrangement of the contacts was suitable for sensitive five-probe measurements [19].

\section{Experimental results and discussion}

Figures $2 \mathrm{a}$ and $\mathrm{b}$ present conductance changes as a function of the magnetic field perpendicular to the wires for $\mathrm{CdTe}: \mathrm{In}$ and $\mathrm{Cd}_{0.99} \mathrm{Mn}_{0.01} \mathrm{Te}$ :In with the electron concentration $9.2 \times 10^{17}$ and $8 \times 10^{17} \mathrm{~cm}^{-3}$, respectively. In both wires weak-field magnetoresistance is observed. In the case of nonmagnetic CdTe wire the magnetoresistance is negative, as it is commonly observed for doped semiconductors, in which cyclotron energy is greater than the spin-splitting. As can be 
seen, the presence of $1 \%$ of magnetic ions changes dramatically the magnetoresistance. Its magnitude is much greater, and below $\approx 4.2 \mathrm{~K}$, becomes positive. As demonstrated previously [18] this positive magnetoresistance is caused by the effect of the giant spin-splitting upon electron-electron interaction. Results of a theoretical computation that takes this effect into account are shown in Fig. 2b by the dashed lines.
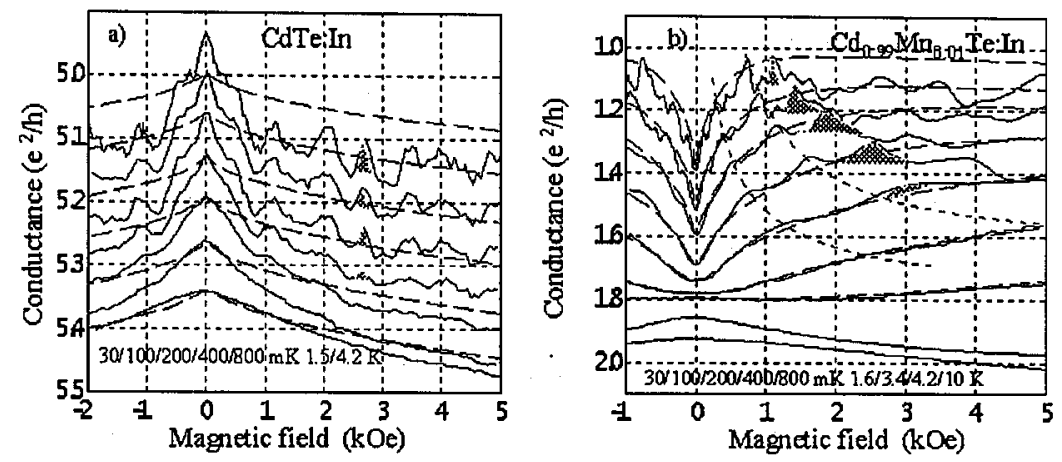

Fig. 2. Conductance $G$ changes as a function of the magnetic field for the wires of $n-\mathrm{Cd}_{1-x} \mathrm{Mn}_{x} \mathrm{Te}$ with $x=0$ (a) and $x=0.01$ (b) at various temperatures. Dashed lines represent magnetoresistance calculated in the framework of $3 \mathrm{D}$ weak-localization theory [18]. Dotted lines visualize a strong temperature dependence of the resistance features in $\mathrm{CdMnTe}(\mathrm{b})$. Note that $G$ decreases in upward direction.

In both materials, the non-periodic and reproducible conductance fluctuations are seen. In the case of the magnetic wire, however, their character is unusual. As shown in Fig. 2b by dotted lines and shadowed areas, the characteristic points of the fluctuation pattern shift towards higher magnetic field with increasing temperature. At the same time, the correlation field $B_{\mathrm{c}}$, which characterizes a typical width of the fluctuation features, increases with either temperature or the magnetic field. Such a behavior is not observed in the case of nonmagnetic wires, including those of $n$-CdTe. This new effect does not depend on the orientation of the magnetic field with respect to the wire, which suggests its spin rather than orbital origin. These findings have led us to propose [6] the existence of new driving. mechanism of UCF. In contrast to normally observed orbital (or Aharonov-Bohm type) effect, in which according to Eq. (3), the magnetic field induces UCF by changing the relative phase of the Feynman paths, the effect observed in CdMnTe has its origin in the spin-splitting-induced redistribution of the carriers between the spin subbands. The redistribution, and the corresponding shift of the Fermi energy $\varepsilon_{F}$ with respect to the bottom of the spin-down and spin-up subbands, changes the wave vector $k_{\mathrm{F}}$ at the Fermi level, as it is shown in Fig. 3a. According to the first term in Eq. (3), this induces the conductance fluctuations. Since the spin-splitting is proportional to the magnetization, the fluctuation pattern should scale with magnetization $M$, not with the magnetic field $B$. That this is indeed the case is shown in Fig. $3 \mathrm{~b}$. 

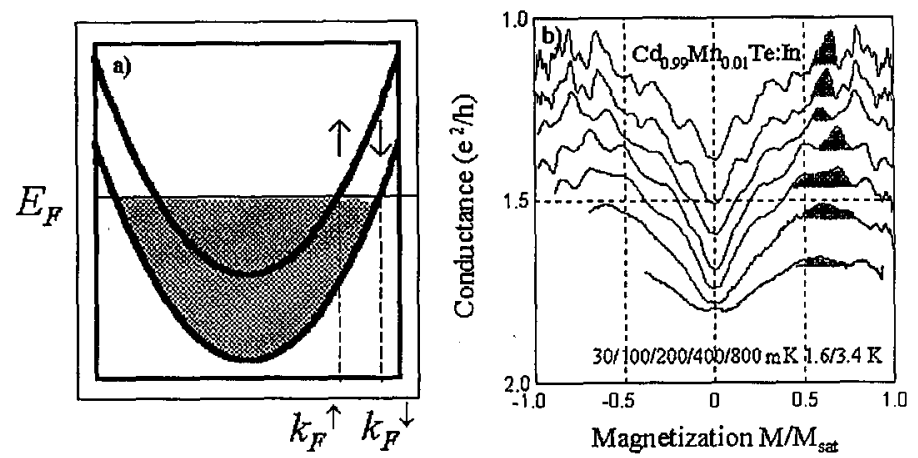

Fig. 3. Schematic view of energy levels in semiconductor with large spin-splitting. Arrows denote spin-up and spin-down subbands (a). Magnetoresistance data for CdMnTe plotted as a function of magnetization (b).
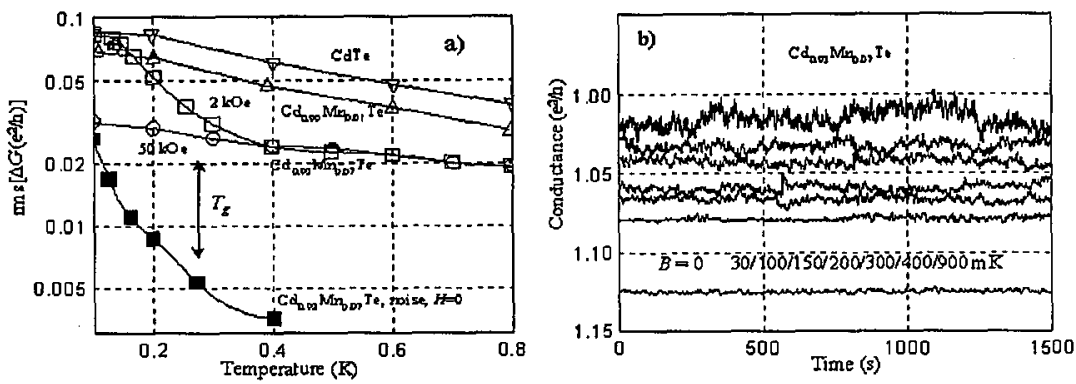

Fig. 4. Conductance of the $\mathrm{Cd}_{0.93} \mathrm{Mn}_{0.07} \mathrm{Te}$ wire with $n=5 \times 10^{18} \mathrm{~cm}^{-3}$ as a function of the magnetic field (a) and time (b) at selected temperatures from $30 \mathrm{mK}$ (top curve) to $900 \mathrm{mK}$ (bottom curve) measured at $I=0.5 \mathrm{nA}$. (c) Temperature dependencies of the root mean square conductance fluctuations.

As depicted in Fig. 4a, the root mean square amplitude of the conductance fluctuations increases with decreasing temperature according to $\operatorname{rms}(\Delta G) \propto T^{-1 / 2}$ for $\mathrm{CdTe}$ and $\mathrm{Cd}_{0.99} \mathrm{Mn}_{0.01} \mathrm{Te}$. While such a behavior is typical of nonmagnetic $1 \mathrm{D}$ wires $[1,20,21]$, it comes as a surprise in the case of $\mathrm{Cd}_{0.99} \mathrm{Mn}_{0.01} \mathrm{Te}$. Indeed, spin-flip scattering, as an inelastic process should destroy coherence of electron waves. Thus $\operatorname{rms}(\Delta G)$ is expected to be controlled by spin-flip scattering rate, $\tau_{\mathbf{s}}$, which for $T>T_{0}$ is independent of temperature but increases with the magnetic field $[22,23]$. However, because of the low density of states specific to semiconductors, we find $\tau_{\mathrm{s}}^{-1}$ to be as low as $10^{10} \mathrm{~s}^{-1}$ at $H=0$. This estimate explains the minor importance of spin-disorder scattering in the considered sample with the low content of the $\mathrm{Mn}$ ions. In contrast, in the case of the $\mathrm{Cd}_{0.93} \mathrm{Mn}_{0.07} \mathrm{Te}$ wire, $\operatorname{rms}(\Delta G)$ is weakly temperature dependent and smaller than that in the wires with $x \leq 0.01$ in the low magnetic field region and at $T>0.3 \mathrm{~K}$. However, $\operatorname{rms}(\Delta G)$ is seen to increase abruptly below $0.3 \mathrm{~K}$, a value corresponding to spin-glass freezing temperature $T_{\mathrm{g}}$ in the bulk material with $x=0.07$. As is seen in Fig. $4 \mathrm{~b}$, in the same temperature range, a dramatic increase in the conductance noise is observed. The presence of noise means that the conductance varies as a 
function of time, contrary to the previously discussed conductance fluctuations, which were non-periodic but fully reproducible conductance changes as a function of the magnetic field. Our findings reveal, therefore, a destructive effect of the fluctuating spins in paramagnetic phase on the UCF, and the appearance of the low frequency noise when the spin dynamics become slow, corroborating qualitatively expectations of theoretical models [11,23] and numerical simulations [17].

As shown in Figs. 5 and 6, particularly strong and complex signatures of the spin-glass freezing are found in the case of $n^{+}-\mathrm{Cd}_{0.8} \mathrm{Mn}_{0.2} \mathrm{Te}$ wire. Such a
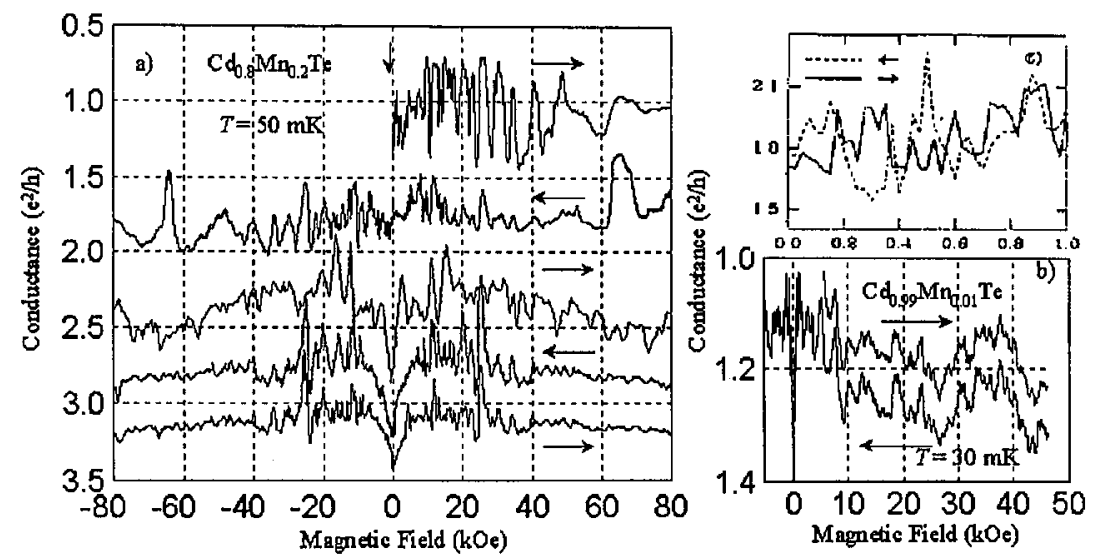

Fig. 5. Conductance $G$ as a function of the magnetic field observed for the wire of $\mathrm{Cd}_{0.8} \mathrm{Mn}_{0.2} \mathrm{Te}(\mathrm{a}), \mathrm{Cd}_{0.99} \mathrm{Mn}_{0.01} \mathrm{Te}$ (b) at $50 \mathrm{mK}$ and calculated [17] by Monte Carlo simulations (c). The traces in (a) and (b) are shifted for clarity. Arrows indicate the directions of sweeps of the magnetic field.
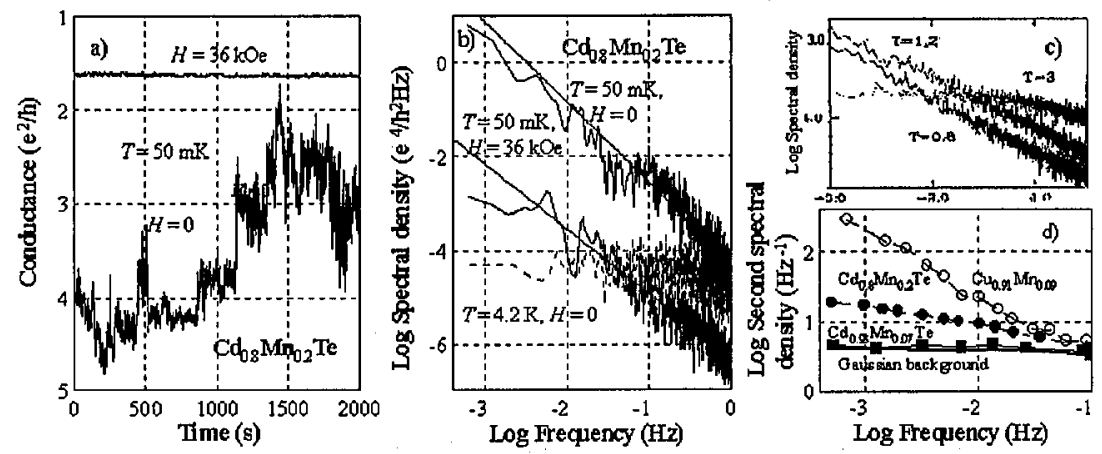

Fig. 6. Conductance $G$ as a function of time in the wire of $n^{+}{ }_{-} \mathrm{Cd}_{0.8} \mathrm{Mn}_{0.2} \mathrm{Te}$ at $50 \mathrm{mK}$ for $B=0$ and $B=3.6 \mathrm{~T}$ (a). Fourier power spectra of noise, $S(f)$ at selected temperatures and magnetic fields from the experiment (b) and as predicted by Monte Carlo simulations [17] (c). The frequency dependence of normalized to Gaussian background second spectral density $s^{2}(f)$ is shown in (d) for $\mathrm{Cd}_{0.93} \mathrm{Mn}_{0.07} \mathrm{Te}$ and $\mathrm{Cd}_{0.8} \mathrm{Mn}_{0.2} \mathrm{Te}$ at $50 \mathrm{mK}$ and $\mathrm{Cu}_{0.91} \mathrm{Mn}_{0.09}$ at $11 \mathrm{~K}$ [13]. Lines in (b) show $1 / f^{\gamma}$. 
sensitivity of the conductance to spin configurations in the whole temperature range below $1 \mathrm{~K}$ stems from a large value of the freezing temperature $T_{\mathrm{g}} \approx 2.2 \mathrm{~K}$ in this material as well as from the proximity to the metal-to-insulator transition. As shown in Fig. 5a, we observe large irreversibilities during cycling between high fields $\pm B_{\mathrm{m}}=8 \mathrm{~T}$ and at $T=50 \mathrm{mK}$, a factor 50 lower than the $T_{\mathrm{g}}$. Magnetotraces were taken after a heat pulse and cooling from $T=5 \mathrm{~K}$. The effect of aging, that is, a gradual decrease in both the fluctuation amplitude and the differences between subsequent traces is clearly visible. As shown in Fig. 5b, c, such conductance irreversibilities were predicted by Monte Carlo simulations, and are absent in the paramagnetic phase.

As shown in Fig. 6a, the conductance noise of the $n^{+}-\mathrm{Cd}_{0.8} \mathrm{Mn}_{0.2} \mathrm{Te}$ wire is also very strong. According to spectral densities $S(f)$ presented in Fig. 6b, the noise is white at $T>T_{\mathrm{g}}$, i.e. its amplitude does not depend on the frequency. Below $T_{\mathrm{g}}$, however, $S(f)$ is seen to assume the form $1 / f^{\gamma}$, where at $50 \mathrm{mK}$ and in $B=0, \gamma=1.3$ and 1.5 for $x=0.07$ and 0.2 , respectively.

An important aspect of our data is that they provide information on how the magnetic field affects the spin-glass dynamics. It may appear that the field will reduce fluctuations of the $\mathrm{Mn}$ spins and, thus, will result in an increase in the UCF amplitude. Indeed, such an increase has been found by others [15] and by us in the paramagnetic phase. At $T<T_{\mathrm{g}}$, however, $\operatorname{rms}(\Delta G)$ of both the UCF (Fig. 4a) and noise (Fig. 6a) is observed to decrease when the magnetic field gets stronger. This observation leads to conclusion that the external magnetic field shifts the spectral weight of the magnetic excitations toward higher frequencies, i.e. drives spin glass toward the paramagnetic phase.

It is still an open question whether spin-glass dynamics is more accurately described in terms of spin droplet excitations [16] or by hierarchical dynamics [24]. These models assumes the short- and long-range coupling between the localized spins, respectively. Both models expect $1 / f$ noise of magnetization, due to a wide spectrum of thermally activated excitations. The first model predicts the existence of a unique ground states, and assigns the glassy dynamics to the thermal generation and subsequent annihilation of independent droplets with reversed spins. In the case of small samples the corresponding noise would come from few fluctuators (droplets) characterized by time independent characteristic frequencies. According to the other model, the spin system wanders between many possible ground states. The particular states have almost identical energies but are separated by very different energy barriers. This results in a broad spectrum of times corresponding to transitions between the particular free energy minima. In the case of small samples the corresponding noise comes again from few fluctuators, whose characteristic frequencies wander, however, with time leading to strong non-Gaussian effects. In macroscopic samples these effects are washed out according to central-limit theorem, since large number of non-Gaussian processes always averages out to a Gaussian one.

It is not easy to measure magnetization noise in small samples directly, because of limited sensitivity of the SQUID magnetometry. Extreme sensitivity of conductance to the instantaneous shape of scattering potential in mesoscopic regime may serve as a powerful tool to study spin dynamics. Statistical analysis 
of the non-Gaussian effects of noise [13] makes it possible to discriminate between these two basic models of the spin glasses. To detect non-Gaussian effects we employed method of the so-called second spectral density. The first power spectral density is $S\left(f_{n}\right)=A_{n} A_{n}$, where $A_{n}$ is the Fourier coefficient of the $n$-th discrete frequency. Accordingly, the second spectral density, is a higher, fourth-order statistic: $S^{2}\left(f_{p}\right)=\sum_{k} \sum_{n} A_{k+p} A_{k}^{*} A_{n+p}^{*} A_{n}$. As is seen, if there were no correlation in the measured signal, $S^{2}$ would be proportional to square of the first spectrum. It has been shown $[13,25,26]$ that if the second spectrum is flat, i.e., its magnitude does not depend on frequency, the relevant fluctuators are uncorellated as in the case of the droplet excitations. In contrast, $S^{2} \propto 1 / f^{\delta}$ with the exponent $\delta \approx 1$ means that noise comes from the pool of wandering events. Actually, $\delta=1-\beta$, where $\beta$ describes the probability of return of the system to previous ground state. In the case of droplet model we deal with one unique ground state and $\beta \equiv 1$. In turn, in hierarchical dynamics the system never comes back to the same ground state, and $\beta=0$.

As is shown in Fig. 6d, we found $\delta=0$ and 0.3 for the case of $\mathrm{Cd}_{1-x} \mathrm{Mn}_{x} \mathrm{Te}$ with $x=0.07$ and 0.2 , respectively. This means that in the case of our wires the second spectra are much flatter than the first ones. Even in the case of $x=0.2$ wire, $\delta \approx 0.3 \approx 0.2 \gamma$, while in metallic spin glass CuMn [13] it was found $\delta \approx 0.9 \gamma$. Thus, in contrast to the latter the data for $\mathrm{Cd}_{0.8} \mathrm{Mn}_{0.2} \mathrm{Te}$ are not consistent with the model of hierarchical dynamics.

\section{Conclusion}

The millikelvin studies of mesoscopic systems discussed in the present paper led to the discovery of a new driving mechanism of UCF in magnetic systems, the spin-splitting induced redistribution of the carriers between the spin subbands. At the same time, several manifestations of spin-glass freezing were detected, recovery of the UCF amplitude below the freezing point, magnetic irreversibilities, aging, and $1 / f$ conductance noise. The statistical analysis of the conductance time series indicated that in contrast to diluted magnetic metals [13], DMS constitute the material system, to which the description of spin-glass properties in terms of droplet excitations [16] may directly apply.

\section{Acknowledgments}

I would like to thank T. Dietl for his guidance through mesoscopic physics. I am also thankful to G. Karczewski, T. Wojtowicz, and J. Kossut, as well as to J. Wróbel, M. Sawicki, T. Skośkiewicz, E. Kamińska, E. Papis, and A. Piotrowska for their contribution to fabrication of DMS quantum wires and for many valuable discussions and comments. Also, I would like to thank W. Plesiewicz for cryogenic expertise. The work was supported under contracts No. 2-P03B-06411 and -11914, and PBZ-28.11 of the Committee for Scientific Research.

\section{References}

[1] For a review on mesoscopic phenomena, see Mesoscopic Phenomena in Solids, Eds. B.L. Altshuler, P.A. Lee, R.A. Webb, Amsterdam, Elsevier 1991; Y. Imry, Introduction to Mesoscopic Physics, Oxford University Press, New York 1997. 
[2] B.L. Altshuler, Pis'ma Zh. Eksp. Teor. Fiz. 51, 530 (1985) [JETP Lett. 41, 648 (1985)].

[3] A.D. Stone, Phys. Rev. Lett. 54, 2692 (1985).

[4] S. Feng, P. Lee, A.D. Stone, Phys. Rev. Lett. 56, 1960 (1986).

[5] R.P. Feynman, R.B. Leighton, M. Sands, The Feynman Lectures on Physics, Addison-Wesley, Reading 1965, Part I, Ch. 37.

[6] J. Jaroszyński, J. Wróbel, M. Sawicki, E. Kamińska, T. Skośkiewicz, G. Karczewski, T. Wojtowicz, A. Piotrowska, J. Kossut, T. Dietl, Phys. Rev. Lett. 75, 3170 (1995).

[7] J. Jaroszyński, J. Wróbel, G. Karczewski, T. Wojtowicz, T. Dietl, Phys. Rev. Lett. 80, 5635 (1998).

[8] For a review on DMS, see e.g. T. Dietl, in: Handbook on Semiconductors, Ed. T.S. Moss, Vol. 3b, North-Holland, Amsterdam 1994, p. 1251; J.K. Furdyna, J. Appl. Phys. 64, R29 (1988).

[9] See, J.A. Mydosh, Spin Glasses: An Experimental Introduction, Taylor \&Francis, London 1993.

[10] M.A. Novak, O.G. Symko, D.J. Zheng, S. Oseroff, J. Appl. Phys. 57, 3418 (1985);

R.R. Gałązka, S. Nagata, P.H. Keesom, Phys. Rev. B 22, 3344 (1980).

[11] B.L. Altshuler, B.Z. Spivak, Pis'ma Zh. Eksp. Teor. Fiz. 42, 363 (1985) [JETP Lett. 42, 477 (1986)].

[12] S. Feng, A.J. Bray, P.A. Lee, M.A. Moore, Phys. Rev. B 36, 5624 (1987).

[13] M.B. Weissman, N.E. Israeloff, G.B. Alers, J. Magn. Magn. Mater. 114, 87 (1992); M.B. Weissman, Rev. Mod. Phys. 65, 829 (1993).

[14] G. Neuttiens, C. Strunk, C. Van Haesendonck, Y. Bruynseraede, Phys. Rev. B, in press.

[15] A. Benoit, D. Mailly, P. Perrier, P. Nedellec, Superlattices Microstruct. 11, 313 (1992).

[16] D.S. Fisher, D.A. Huse, Phys. Rev, B 38, 373, 386 (1988).

[17] M. Cieplak, B.R. Bułka, T. Dietl, Phys. Rev. B 44, 12337 (1991); ibid. 51, 8939 (1995).

[18] M. Sawicki, T. Dietl, J. Kossut, J. Igalson, T. Wojtowicz, W. Plesiewicz, Phys. Rev. Lett. 56, 508 (1986).

[19] J. Scofield, Rev. Sci. Instrum. 58, 985 (1987).

[20] B.L. Altshuler, B.I. Shklovskii, Zh. Eksp. Teor. Fiz. 91, 220 (1986) [Sov. Phys.-JETP 64, 127 (1986)]; P.A. Lee, A.D. Stone, H. Fukuyama, Phys. Rev. $B$ 35, 1039 (1987).

[21] See, C.V.J. Beenakker, H. Van Houten, Solid State Phys. 44, 1 (1991).

[22] A. Benoit, S. Washburn, C.P. Umbach, R.A. Webb, D. Mailly, L. Dumoulin, in: Anderson Localization, Eds. T. Ando, H. Fukuyama, Springer, Berlin 1988, p. 346.

[23] A.A. Bobkov, V.I. Falko, D.E. Khmelnitskii, Zh. Eksp. Teor. Fiz. 98, 703 (1990) [Sov. Phys.-JETP 71, 393 (1990)]; V.I. Falko, J. Phys., Condens. Matter 4, 3943 (1992).

[24] G. Parisi, Phys. Rev. Lett. 43, 1754 (1979).

[25] G.T. Seidler, S.A. Solin, Phys. Rev. B 53, 9753 (1996).

[26] K.M. Abkemeier, Phys. Rev. B 55, 7005 (1997). 